\title{
A comparative assessment of different methods for Landsat 7/ETM+ pansharpening
}

\author{
VICTOR F.RODRIGUEZ-GALIANO* †, EULOGIO PARDO-IGÚZQUIZA \\ MARIO CHICA-OLMO $\dagger$, JAVIER MATEOS $\S$, JUAN P. RIGOL-SÁNCHEZ $\uparrow$ and \\ MIGUEL VEGA|
}

\begin{abstract}
†Dept. de Geodinámica, Universidad de Granada, Granada 18071, Spain ‡Instituto Geológico y Minero de España (IGME), Madrid 28003, Spain $\S$ Dept. de Ciencias de la Computación e I. A., Universidad de Granada, Granada 18071, Spain

đDept. de Geología, Universidad de Jaén, Jaén 23071, Spain

|Dept. de Lenguajes y Sistemas Informáticos, Universidad de Granada, Granada 18071, Spain
\end{abstract}

*Corresponding author. Email: vrgaliano@ugr.es

\begin{abstract}
The present paper compares a set of relevant methods, based on different mathematical approaches, for Landsat $7 \mathrm{ETM}+$ pansharpening. These are classical procedures such as Principal Component Analysis (PCA), Intensity Hue Saturation (FIHS); based on wavelet transforms: Wavelet à Trous (WAT), Additive Wavelet Luminance Proportional method (AWLP) and Multidirection-Multiresolution (MDMR); a method of a geostatistical nature, Downscaling Cokriging (DCK); and finally, a Bayesian method (L1COR).

The comparison of the fused images is based on the qualitative and quantitative evaluation of their spatial and spectral characteristics by calculating statistical indexes and parameters that measure the quality and coherence of the images. Moreover, the quality of the spectral information is studied indirectly, by means of the ISODATA classification of the products of fusion.

The results shows that DCK and L1COR methods yielded better results than the wavelet based did. Particularly, DCK does not introduce artefacts in the estimation of the digital numbers corresponding with the source multispectral image and, therefore, it can be considered as the most coherent method.
\end{abstract}

\section{Keywords}

\section{Image Enhancement, Pansharpening, ETM+, Wavelet Transforms, Geostatistics, Bayesian Approach}

\section{Introduction}

The arrival of new sensors and satellites in recent decades has notoriously favoured the availability of remotely sensed images with enhanced spatial and spectral resolution. In practice, a more effective use of this information entails the application of image fusion techniques in order to obtain a final product with spatial and spectral resolution characteristics suitable for a specific application. Typically, high spatial resolution panchromatic images are integrated with high spectral resolution multispectral images. 
Methodologies for image fusion comprise a set of procedures with diverse conceptual bases. They range from techniques based on simple arithmetic operations to complex algorithms based on the application of convolutions and filters.

The simplest fusion techniques even carry out the direct substitution of some bands for visualization, or just a simple arithmetic transformation is applied, as in the Brovey method (Ehlers 1991). Some other classical image fusion algorithms are more complex and involve transformations of the images and substitution of components, e.g. Principal Component Analysis (PCA), IHS (Buchanan and Pendergrass 1980, Carper et al. 1990). In these methods, an intensity image is produced and then replaced in the inverse transformation by a high spatial resolution panchromatic image that is considered equivalent.

Over the last decade, wavelet transforms have been applied to fuse multispectral and panchromatic satellite imagery (Garguet-Duport et al. 1996, Ranchin and Wald 2000, Aiazzi et al. 2002, Simone et al. 2002, Ranchin et al. 2003, Amolins et al. 2007). These fusion algorithms may be considered as an extension of the High Pass Filtering (HPF) method (Chavez et al. 1991), since they hold that spatial information is contained in the high frequencies. The wavelet transforms extract detailed information of the panchromatic image to integrate it subsequently into the multispectral image by using substitution methods, addition methods or a selection of methods based on the frequency or the spatial context. An additional advantage of these techniques is that the wavelet function can be modified to present specific features useful for a particular application of the transformation (Mallat 1999, Lillo-Saavedra and Gonzalo 2006, Amolins et al. 2007, Lillo-Saavedra and Gonzalo 2007).

Fusion approaches based on the Bayesian modelling and inference, have recently been proposed. These approaches incorporate prior knowledge on the expected characteristics of the multispectral images, model the observation process of both panchromatic and multispectral images and apply inference methods to estimate the distribution of the high spatial resolution multispectral image (Molina et al. 2008, Vega et al. 2010). Vega et al. (2010) proposed a Bayesian method, L1COR, which considers the correlation between the multispectral bands themselves, besides considering the relationship between the panchromatic image and the multispectral band. This is a desirable property, as it has been shown that the multispectral bands of certain sensors (i.e. Landsat $7 \mathrm{ETM}+$ ) are only partially covered by the panchromatic image.

A methodological alternative to the abovementioned is grounded on geostatistical methods, which, unlike those presented previously, explicitly account for the spatial variability characteristics of the images to be fused (Nishii et al. 1996, Chica-Olmo and Abarca-Hernandez 1998, Memarsadeghi et al. 2005). The geostatistical fusion model is based on the cokriging method (Atkinson et al. 1992, 1994, P. and Delfiner 1999). A variant of this methodology is the Downscaling Cokriging method (DCK), proposed by (Pardo-Iguzquiza et al. 2006), which considers relevant aspects for image fusion such as pixel size (information support), the direct and cross-spatial correlations of the digital values of the images, and the point spread functions of the sensors.

Although several comparative studies on remote sensing image fusion methods have been published, only a few include a detailed assessment of the results obtained with a broad range of available techniques. Thus, (Chavez et al. 1991), (Ehlers 1991) and 
(Rigol and Chica-Olmo 1998) have compared results based on classical methods such as BR, PCA, IHS, and HPF. Likewise, (Yocky 1996), (Garguet-Duport et al. 1996), and (Pohl and Van Genderen 1998, Ranchin and Wald 2000) carried out studies comparing classical techniques with wavelet transforms. Alparone et al. (2007) tested different algorithms of different philosophies to merge QuickBird and simulated Pléiades.

The goal of this research paper is to perform a detailed comparative assessment of a set of relevant image fusion techniques for Landsat 7/ETM+ pansharpening. In particular, a detailed comparative analysis of seven image fusion algorithms, representative of different methodological approaches, is presented: two classical methods, Principal Component Analysis (PCA) and Intensity Hue Saturation (FIHS); a Bayesian method (L1COR); a geostatistical method, Downscaling Cokriging (DCK); and, lastly, three methods based on wavelet transforms, such as Wavelet à Trous (WAT), Additive Wavelet Luminance Proportional (AWLP) and Multidirection-Multiresolution (MDMR).

The study is illustrated using a multispectral Landsat 7 Enhanced Thematic Mapper $(\mathrm{ETM}+)$ image and its corresponding panchromatic image, with a spatial resolution of $30 \mathrm{~m}$ and $15 \mathrm{~m}$, respectively. The image was acquired on 20 July 2002 over the metropolitan area of Granada, in southeast Spain. The scene corresponds to path 200 row 34 of the Landsat Worldwide Reference System (WRS). A sector of the Landsat scene of $944 \mathrm{~km}^{2}(1024 \times 1024$ pixels of $30 \mathrm{~m} \mathrm{x} \mathrm{30m)} \mathrm{was} \mathrm{selected} \mathrm{for} \mathrm{the} \mathrm{analysis}$ (figure 1), which includes urban areas and small agricultural lots.

\section{[Insert Figure 1. Approximately here]}

The results of the fusion have been evaluated from different perspectives (Wald et al. 1997, Ranchin et al. 2003): qualitatively, judging the visual quality of the image; and quantitatively, by degrading all available data to a coarser resolution, carrying out fusion from such data and calculating indexes and statistics of the spectral and spatial quality. Moreover, another experiment has been carried out to calculate "coherence measures" by degrading the fused images to its original resolution. Finally, the spectral quality of the fusion has also been assessed indirectly through the digital classification of the fused image.

\section{A Brief Review of Fusion Methods}

In this section, we briefly describe the seven fusion algorithms that have been chosen for comparative study. These algorithms present a different computational and application complexity:

\subsection{PCA Method}

The PCA method is based on the application of a classical statistical procedure of principal component analysis of the original bands of the multispectral image. According to Chávez et al. (1991), in the calculation process of the principal components, most of the common information of the set of multispectral bands is contained in the first component. This component is substituted by the panchromatic 
band, equivalent in load of radiometric information, but with a better spatial resolution. The inverse transformation allows obtaining the fused image.

\subsection{FIHS Method}

The IHS is probably the fusion algorithm most often used in remote sensing applications. It is based on the colour space transformation from RGB to IHS, and substitution of the resulting intensity band by the high spatial resolution panchromatic image (Carper et al. 1990, Ehlers 1991, Shettigara 1992, Grasso 1993, Pellemans and Jordans 1993). By applying the inverse transformation after substitution, a multispectral image similar to the initial one, but with an enhanced spatial resolution is obtained. The FIHS fusion algorithm is based on the same theoretical grounds as the IHS, but the inverse transformation process is simplified (Tu et al. 2005). The main advantage of this algorithm with respect to IHS is that it can be applied to any number of bands, not just to a band triplet.

\subsection{L1COR Method}

The Bayesian formulation of the pansharpening problem starts with the definition of the joint distribution $p(\mathbf{y}, \mathbf{Y}, \mathbf{x})$, where $\mathbf{y}$ is the unknown high resolution multispectral image which has $M$ bands $\mathbf{y}_{k}, k=1, \ldots, M, \mathbf{Y}$ is the observed low resolution multispectral image and $\mathbf{x}$ is the panchromatic image. This joint distribution can be defined as $p(\mathbf{y}, \mathbf{Y}$, $\mathbf{x})=p(\mathbf{y}) \mathrm{p}(\mathbf{Y}, \mathbf{x} \mid \mathbf{y})$ and inference is based on $p(\mathbf{y} \mid \mathbf{Y}, \mathbf{x})=p(\mathbf{y}) p(\mathbf{Y}, \mathbf{x} \mid \mathbf{y}) / p(\mathbf{Y}, \mathbf{x})$.

The L1COR method (Vega et al. 2010) proposes a prior model based on the L1 norm to take into account the smoothness within each band plus an auto-regressive model to exploit the correlation between the image bands, thus defining the multispectral image prior:

$$
p(\mathbf{y}) \propto \exp \left\{\sum_{\substack{k, k^{\prime}=1 \\ k^{\prime}>k}}^{M} \frac{v_{k k^{\prime}}}{2}\left\|\mathbf{y}_{b}-\mathbf{y}_{k^{\prime}}\right\|^{2}\right\} \prod_{k=1}^{M} \exp \left\{-\sum_{i=1}^{P}\left[\alpha_{k}^{h}\left\|\Delta_{i}^{h}\left(\mathbf{y}_{k}\right)\right\| 1+\alpha_{k}^{v}\left\|\Delta_{i}^{v}\left(\mathbf{y}_{k}\right)\right\|_{1}\right]\right\}
$$

where the quadratic terms enforce similarity between the pixels in the same position in the different bands with $v_{k k^{\prime}}>0$ being the parameter that control the similarity between band $k$ and band $k^{\prime}$ and the L1 norm priors impose smoothness into each multispectral band. In the previous expression, $\Delta_{i}^{h}\left(\mathbf{y}_{k}\right)$ and $\Delta_{i}^{v}\left(\mathbf{y}_{k}\right)$ represent the horizontal and vertical first order differences at pixel $i$, respectively, $p$ the number of pixels of the high resolution images, and $\alpha_{k}^{h}$ and $\alpha_{k}^{v}$ are the horizontal and vertical model parameter for the band $k$. The L1 model enforces smoothness within the objects in each image band, controlling the noise while preserving their edges whereas the quadratic model enforces spectral smoothness.

Since the observed panchromatic and low resolution multispectral images are independent given the real high resolution multispectral image to be estimated, we can 
write $p(\mathbf{Y}, \mathbf{x} \mid \mathbf{y})=p(\mathbf{Y} \mid \mathbf{y}) p(\mathbf{x} \mid \mathbf{y})$. The conditional distribution of the observed multispectral image, $\mathbf{Y}$, given the real high resolution multispectral image, $\mathbf{y}$, is given by

$$
p(\mathbf{Y} \mid \mathbf{y}) \propto \prod_{k=1}^{M} \exp \left\{-\frac{1}{2} \beta_{k}\left\|\mathbf{Y}_{k}-\mathbf{H} \mathbf{y}_{k}\right\|^{2}\right\}
$$

where $\beta_{k}$ is the inverse of the variance of the independent Gaussian noise in each band $k$, and $\mathbf{H}=\mathrm{DB}$, with $\mathbf{B}$ a blurring matrix which takes into account the sensor integration function and any other blur that may degrade the image and $\mathrm{D}$ a decimation operator that match the fusion ratio.

The conditional distribution of the panchromatic image $\mathbf{x}$ given $\mathbf{y}$, is defined as

$$
p(\mathbf{x} \mid \mathbf{y}) \propto \exp \left\{-\frac{1}{2} \gamma\left\|\mathbf{x}-\sum_{k=1}^{M} \lambda_{k} \mathbf{y}_{k}\right\|^{2}\right\}
$$

where $\gamma$ is the inverse of the zero mean Gaussian noise of the panchromatic image and $\lambda_{k} \geq 0$ are known quantities that weight the contribution of each high spatial resolution multispectral band $y_{k}$ to the panchromatic image, $\mathbf{x}$. The values can be calculated, as we will see later, from the spectral response of the sensor.

Once the parameters are known or previously estimated (Molina et al. 2008, Vega et al. 2010), the Bayesian paradigm dictates that inference on $\mathbf{y}$ should be based on the posterior distribution $p(\mathbf{y} \mid \mathbf{Y}, \mathbf{x})$. Since $p(\mathbf{y} \mid \mathbf{Y}, \mathbf{x})$ cannot be found in closed form, variational methods are applied to approximate this distribution by another distribution, $q(\mathbf{y})$, that minimizes the Kullback-Leibler divergence of $p(\mathbf{y} \mid \mathbf{Y}, \mathbf{x})$ and $q(\mathbf{y})$ and that make the inference process tractable. The estimated high resolution multispectral image can be obtained as the mean of the distribution $q(\mathbf{y})$.

\subsection{DCK Method}

The fused image of high spatial resolution obtained through the Downscaling Cokriging method can be expressed as a linear combination of the experimental images (PardoIguzquiza et al. 2006, Atkinson et al. 2008, Pardo-Iguzquiza et al. 2011):

$$
\hat{Z}_{u_{0}}^{k_{0}}\left(\boldsymbol{x}_{0}\right)=\sum_{j=1}^{M} \sum_{i=1}^{n_{j}} \lambda_{j i}^{0} Z_{u_{j}}^{k_{j}}\left(\boldsymbol{x}_{i}\right)
$$

Where:

$Z_{a}^{b}(\boldsymbol{x})$ : represents the digital number of a satellite image for the spectral band $b$ and with a spatial resolution (pixel size) $a$ and at a particular spatial location $\boldsymbol{x}=\{x, y\}$. The circumflex symbol above $\mathbf{Z}$ denotes that it is an estimated or fused image by cokriging, whereas without the circumflex accent it is designated as an experimental image. Other annotations are:

$b=k_{0}$ spectral band whose spatial resolution should be improved. 
$b=k_{j}$ experimental spectral band included in the process of fusion by cokriging.

$a=u_{0}$ spatial resolution or pixel size of the fused image.

$a=u_{j}$ spatial resolution or pixel size of an experimental image used in the fusion.

$M$ : number of experimental bands used in the fusion.

$n_{j}$ : number of pixels of the neighbourhood used for the experimental image of the spectral band $k_{j}$. Typically, these neighbourhoods are moving windows $3 \times 3,5 \times 5,7 \times$ 7 , etc.

$\lambda_{j i}^{0}$ : optimal weight applied to $Z_{u_{j}}^{k_{j}}\left(\boldsymbol{x}_{i}\right)$ in the estimation of $Z_{u_{0}}^{k_{0}}\left(\boldsymbol{x}_{0}\right)$.

The optimal weights given above are obtained by means of the resolution of a system of linear equations known as a cokriging system. This system is derived by imposing that the estimator be unbiased:

$$
\mathrm{E}\left\{\hat{Z}_{u_{0}}^{k_{0}}\left(\boldsymbol{x}_{0}\right)-Z_{u_{0}}^{k_{0}}\left(\boldsymbol{x}_{0}\right)\right\}=0
$$

and minimizing the variance of estimation

$$
\mathrm{E}\left\{\left[\hat{Z}_{u_{0}}^{k_{0}}\left(\boldsymbol{x}_{0}\right)-Z_{u_{0}}^{k_{0}}\left(\boldsymbol{x}_{0}\right)\right]^{2}\right\} \rightarrow \text { minimum }
$$

Where $\mathrm{E}\{$.$\} is the operator of mathematical expectation.$

The cokriging system is represented in the form of a matrix, as:

$$
\left[\begin{array}{cccccc}
\mathbf{C}_{u_{1} u_{1}}^{k_{1} k_{1}} & \cdots & \mathbf{C}_{u_{1} u_{M}}^{k_{1} k_{M}} & \mathbf{1}_{n_{1}} & \cdots & \boldsymbol{0}_{n_{1}} \\
\vdots & \ddots & \vdots & \vdots & \ddots & \vdots \\
\mathbf{C}_{u_{M} u_{1}}^{k_{M_{1} k_{1}}} & \cdots & \mathbf{C}_{u_{M} u_{M}}^{k_{k_{M}} k_{M}} & \boldsymbol{0}_{n_{M}} & \cdots & \boldsymbol{1}_{n_{M}} \\
\mathbf{1}_{n_{1}}^{t} & \cdots & \boldsymbol{0}_{n_{M}}^{t} & 0 & \cdots & 0 \\
\vdots & \ddots & \vdots & \vdots & \ddots & \vdots \\
\boldsymbol{0}_{n_{1}}^{t} & \cdots & \mathbf{1}_{n_{M}}^{t} & 0 & \cdots & 0
\end{array}\right]\left[\begin{array}{c}
\boldsymbol{\lambda}_{1}^{0} \\
\vdots \\
\boldsymbol{\lambda}_{M}^{0} \\
\mu_{1} \\
\vdots \\
\mu_{M}
\end{array}\right]=\left[\begin{array}{c}
\mathbf{C}_{u_{0} u_{1}}^{k_{0} k_{1}} \\
\vdots \\
\mathbf{C}_{u_{0} u_{M}}^{k_{1} k_{M}} \\
1 \\
\vdots \\
0
\end{array}\right]
$$

Where:

$\mathbf{C}_{u_{i} u_{j}}^{k_{i} k_{j}}$ : matrix $\left(n_{i} \times n_{j}\right)$ of cross-covariance between the $n_{i}$ pixels of spectral band $k_{i}$ with spatial resolution $u_{i}$ and the $n_{j}$ pixels of spectral band $k_{j}$ with spatial resolution $u_{j}$. Notice that a direct covariance is equivalent to the cross-covariance of an image with respect to itself.

$\mathbf{1}_{n_{i}}^{t}:\left(1 \mathrm{x} n_{i}\right)$ vector of ones.

$\boldsymbol{0}_{n_{i}}^{t}:\left(1 \times n_{i}\right)$ vector of zeros.

The super index $t$ denotes a transposed vector.

$\mu_{k}:(k=1, \ldots, M)$ Lagrange multipliers.

A further explanation about the resolution of the cokriging system (equation (7)) can be seen in (Pardo-Iguzquiza et al. 2006) and (Atkinson et al. 2008). This system accounts 
for three key aspects for image fusion: the experimental images pixel size (support effect), the direct and cross-variograms of the radiometric bands, and the point spread functions of the sensor.

\subsection{WAT Method}

Wavelet transforms are considered as a filter bank which, applied to a sequence of levels of image decomposition, divide the signal (e.g. satellite image) into both high and low frequency components (Amolins et al. 2007). When applied at different levels, the decomposition is referred to as multi resolution decomposition.

The Wavelet à Trous transform, or WAT (Nuñez et al. 1999) involves basically the application of a series of consecutive convolutions for different levels of image degradation (Nuñez et al. 1999, Chibani and Houacine 2002, Ranchin et al. 2003, Garzelli et al. 2004, Gonzalez-Audicana et al. 2005). WAT requires an iterative filtering process, in which several degradation filters are used to obtain the wavelet coefficients. Since it is a non-decimated algorithm, the process starts by using an initial filter to which rows and columns of zeros are iteratively inserted between the rows and columns of the filter of the previous iteration until the desired resolution is achieved.

The WAT method, unlike other algorithms such as the pyramidal one by Mallat (Mallat 1999, Gonzalez-Audicana et al. 2005), is characterized by the directional independence of the filtering process, without spatial compression of the different levels of degradation. Therefore, the image for each level of degradation has half the resolution than the previous one, but the same size, so the information contained in each one of the levels is redundant.

The wavelet coefficients $A^{k j}{ }_{u j+n}(\boldsymbol{x})$ are calculated as the difference between two consecutive levels of degradation:

$$
A_{u_{j}+n}^{k_{j}}(\boldsymbol{x})=Z_{u_{j}+n-1}^{k_{j}}(\boldsymbol{x})-Z_{u_{j}+n}^{k_{j}}(\boldsymbol{x})
$$

$\mathrm{Z}^{\mathrm{k}}{ }_{\mathrm{uj}}(x)$ represents the digital number at a pixel at location $\boldsymbol{x}=(x, y)$ belonging to spectral band $\mathrm{k}_{\mathrm{j}}$ of the original image.

Following an additive criterion, if $Z^{k j}{ }_{u j+n}(\boldsymbol{x})$ represents the successive degradations that contain the information of low frequencies of the original multispectral image, and $A^{k j}{ }_{u j+n}(\boldsymbol{x})$ the respective wavelet coefficients that contain the high frequency information, it is possible to obtain a high-resolution fused image $\hat{Z}_{u_{0}}^{k_{0}}(\boldsymbol{x})$ by means of the sum of the low frequencies contained in the degraded multispectral image and the high frequencies extracted from the panchromatic image.

$$
\hat{Z}_{u_{0}}^{k_{0}}(\boldsymbol{x})=Z_{u_{j}+n}^{k_{j}}(\boldsymbol{x})+\sum_{k=1}^{n} A_{u_{j+n}}^{k_{j}}(\boldsymbol{x})
$$




\subsection{AWLP Method}

The additive wavelet luminance proportional method, AWLP (Otazu et al. 2005), is an extension of the Additive Wavelet Luminance (AWL) method (Nuñez et al. 1999) that considers an arbitrary number of bands in the multispectral image instead of three bands (RGB). In the AWLP method, the high frequency information, $\sum_{k=1}^{n} A_{u_{j+n}}^{k_{j}}(\boldsymbol{x})$, is weighted by a coefficient $Z_{u_{j}+n}^{k_{j}} / \sum_{k=1}^{n} Z_{u_{j}+n}^{k_{j}}$ before being added to the corresponding multispectral band. In this way the details introduced in each reconstructed band are proportional to the original radiance values and, so, the spectral signature is maintained.

\subsection{MDMR Method}

The Multidirection-Multiresolution fusion algorithm (Lillo-Saavedra and Gonzalo 2007) is a modification of the WAT method that incorporates directional transforms. It is an algorithm meant to attain an optimal equilibrium between the spectral and the spatial resolutions of the combined images, by means of the application of directional ellipsoidal filters.

The fusion process is similar to the one put forward by the WAT method, as detailed in the following equations:

$$
\begin{gathered}
A_{\theta_{n}}^{k_{j}}(\boldsymbol{x})=Z_{\theta_{n}}^{k_{j}}(\boldsymbol{x})-Z_{\theta_{n}-1}^{k_{j}}(\boldsymbol{x}) \\
\hat{Z}_{u_{0}}^{k_{0}}(\boldsymbol{x})=Z_{\theta_{l}}^{k_{j}}(\boldsymbol{x})+\sum_{k=1}^{l} A_{\theta_{n}}^{k_{j}}(\boldsymbol{x})
\end{gathered}
$$

However, we see that the level of degradation has been replaced by that of the directional filter of orientation $\theta$. Unlike WAT, this is a highly anisotropic algorithm, which allows weighting the desired spatial and spectral resolutions.

(Lakshmanan 2004) adopted equation (12) to introduce a separable approximation to a directional low-pass filter (equations (14) and (15)).

$$
F(x, y)=\left\{\begin{array}{l}
1 \text { if } \frac{(x \cos \theta-y \sin \theta)^{2}}{a^{2}}+\frac{(x \sin \theta+y \cos \theta)^{2}}{b^{2}} \leq 1 \\
0 \text { otherwise }
\end{array}\right.
$$

Where $a$ and $b$ define the scale of the filter, and their ratio defines the ellipse elongation.

$$
f(x, y)=f_{1}(x) \times f_{2}(y)-\alpha x f_{1}(x) \times y f_{2}(y)
$$


Where $\alpha$ is given by $\left(a^{2}-b^{2}\right) \sin (2 \theta) / a^{2} b^{2}$ and $f_{l}(x)$ and $f_{2}(y)$ by:

$$
\begin{aligned}
& f_{1}(x)=\exp \left(-x^{2}\left(\frac{\cos ^{2} \theta}{a^{2}}+\frac{\sin ^{2} \theta}{b^{2}}\right)\right) \\
& f_{2}(y)=\exp \left(-y^{2}\left(\frac{\cos ^{2} \theta}{b^{2}}+\frac{\sin ^{2} \theta}{a^{2}}\right)\right)
\end{aligned}
$$

\section{Fused Images Quality Assessment}

It is expected that an optimal image fusion methodology does not distort the original information (both spatial and spectral), thus allowing the use of fused images in applications such as digital classification or change analysis. In light of this understanding, a detailed analysis on the quality of the results obtained with the four aforementioned methods for image fusion has been carried out. To this end, the following criteria have been applied, based on visual and numerical aspects, the latter by means of indexes and statistical parameters. The general procedure is sketched in figure 2 .

\section{[Insert Figure 2. Approximately here]}

\subsection{Qualitative Assessment}

The quality of the results generated by the different fusion methodologies was evaluated visually. The criteria used were comparison of tone, contrast, saturation, sharpness, and texture of the fused images.

\subsection{Quantitative Assessment}

To assess numerically the quality of the reconstructed multispectral images, both spatial and spectral measures have to be considered. A set of statistical parameters and indexes were calculated to quantify the differences between the spectral information of the fused images $\left(\mathrm{M}_{\mathrm{h}}{ }^{*}\right)$ and the real image that the Landsat ETM+ sensor would observe with the highest resolution $\left(\mathrm{M}_{\mathrm{h}}\right)$ :

-Correlation coefficient (C).

-Root Mean Square Error (RMSE).

-The ERGAS index (Erreur Relative Globale Adimensionnelle de Synthèse) (Wald 2000, Ranchin et al. 2003):

$$
E R G A S_{\text {spectral }}=100 \frac{h}{l} \sqrt{\frac{1}{N} \sum_{k=1}^{N} \frac{\left(R M S E_{\text {spectral }}\left(\mathbf{B}_{k}\right)\right)^{2}}{M_{k}^{2}}}
$$

Where $h / l$ is the ratio between the resolution of the panchromatic image and the multispectral image, $N$ is the number of spectral bands $\left(\mathbf{B}_{k}\right)$ of the fused image, 
$M_{k}$ is the mean value of each spectral band, and RMSE is the Root Mean Square Error calculated between the fused image and the multispectral original image.

The lesser the ERGAS value, the better the quality of the fused image. Wald et al. (1999) establishes experimentally that an ERGAS value $<3$ corresponds to fused images of satisfactory quality.

-The Image Quality Index (Q), proposed by (Wang and Bovik 2002) as an alternative to the Root Mean Square Error models the differences between two given monochromatic images as a combination of three different factors: loss of correlation, luminance distortion, and contrast distortion.

$$
Q=\frac{4 \sigma_{O F} \bar{O} \bar{F}}{\left(\sigma_{O}^{2}+\sigma_{F}^{2}\right)\left[(\bar{O})^{2}+(\bar{F})^{2}\right]}
$$

Where $\bar{O}$ and $\bar{F}$ are the mean of the original and the fused images, respectively, $\sigma^{2}{ }_{O}$ and $\sigma_{F}^{2}$ are the variances, and $\sigma_{O F}$ is the covariance between the original image and the fused image. In order to avoid errors tied to the index spatial dependence, (Wang and Bovik 2002) recommend the use of moving windows of different sizes. In our case, windows measuring 8x8, 16x16, 32x32, 64x64 and $128 \times 128$ have been used, so the resulting index is the mean of all the partial indexes (Gonzalez-Audicana et al. 2005).

-The Structural Similarity index (SSIM) (Wang et al. 2004), a perceptual quality measure that takes into account the luminance, contrast and structure differences, between each band of the reconstructed and original multispectral images.

$$
\operatorname{SSIM}(\mathbf{x}, \mathbf{y})=\frac{\left(2 \mu_{x} \mu_{y}+C_{1}\right)\left(2 \sigma_{x y}+C_{2}\right)}{\left(\mu_{x}^{2}+\mu_{y}^{2}+C_{1}\right)\left(\sigma_{x}^{2}+\sigma_{y}^{2}+C_{2}\right)}
$$

Where $\mathbf{x}$ and $\mathbf{y}$ are two non-negative image signals, $\mu_{x}$ and $\mu_{y}$ are the luminance of images $\mathbf{x}$ and $\mathbf{y}, \sigma_{x y}, \sigma_{x}$ and $\sigma_{y}$ are the covariance and the variances of image $\mathbf{x}$ and $\mathbf{y}$ respectively and, lastly, $C_{1}$ and $C_{2}$ are constants. The SSIM index takes values between -1 and 1 . The closer the SSIM index to one the better the reconstruction, with 1 only being reachable when the two images are identical.

Spatial improvement was assessed by means of the correlation of the high frequency components (HFC) of the fused images $\left(\mathrm{M}_{\mathrm{h}}{ }^{*}\right)$ and the original panchromatic $\left(\mathrm{P}_{1}\right)$.

The "coherence measure" between the degraded fused images $\left(\mathrm{M}_{1}{ }^{*}\right)$ and the original image $\left(\mathrm{M}_{1}\right)$ was obtained by calculating the correlation coefficient $(\mathrm{C})$ and the real errors (Root Mean Square Error, RMSE).

\subsection{Indirect quantitative Assessment}

This criterion is founded on the digital classification of the fused images and the measure of its mapping accuracy (Congalton 1991, Congalton and Green 1999). 


\section{Results and discussion}

The best reference for assessing the quality of the fused image consists, obviously, in knowing the true or real image $\left(\mathrm{M}_{\mathrm{h}}\right)$ which is wished to be obtained via the fusion method (Wald et al. 1997). However, this is not feasible in practice. For this reason, an experiment has been carried out in which the original multispectral $\left(\mathrm{M}_{\mathrm{h}}\right)$ and panchromatic $\left(\mathrm{P}_{\mathrm{h}}\right)$ images have been degraded to resolutions of $120 \mathrm{~m}\left(\mathrm{M}_{1}\right)$ and $30 \mathrm{~m}\left(\mathrm{P}_{1}\right)$, respectively, in order to obtain fused images with a resolution of $30 \mathrm{~m}\left(\mathrm{M}_{\mathrm{h}}{ }^{*}\right)$ (see figure $2)$. Thus the results of fusion $\left(\mathrm{M}_{\mathrm{h}}{ }^{*}\right)$ can be compared to the real image $\left(\mathrm{M}_{\mathrm{h}}\right)$.

In Addition, any fused multispectral image once degraded to its original resolution 1, should be as identical as possible to the original multispectral image $\mathrm{M}_{1}$ (Wald et al. 1997). To measure the coherence between the said images, the $\left(M_{h}{ }^{*}\right)$ images have been degraded to the spatial resolution 1 .

\subsection{Application of the Fusion Algorithms}

\subsubsection{Classical Methods}

The classical image fusion algorithms, PCA and FIHS, are computationally simple algorithms. These methods do not require definition of filters or the study of spatial variability between images, as required in the case of wavelet, Bayesian and geostatistically based procedures.

\subsubsection{L1COR}

The conditional probability distributions in equations 13 and 14 depend on a set of parameters that models the relationship between the high resolution multispectral image and the panchromatic and the low resolution multispectral image. The values of $\lambda_{k}, k=$ $1, \ldots, 4$, in equation 14 were calculated from the spectral response of the ETM+ sensor, following the procedure described in (Vega et al. 2010) producing values equal to $0.0078,0.242,0.2239$, and 0.5263 , for bands one to four, respectively. Note that the panchromatic image almost does not cover band one and so, the value of $\lambda_{1}$ is very low. The matrix $\mathbf{H}$ is obtained mimicking the degradation process by using $0.125 \times 1_{4 \times 4}$ as sensor integration function and downsampling by a factor of 4 by discarding every other three pixels in each direction. Since no noise was added during the degradation process we chose $\beta_{k}=100$ and $\gamma=10$.

The values for the parameters of the prior model were selected as follows. The values for $\alpha_{k}^{h}$ and $\alpha_{k}^{v}$ were determined as $1 / \sum_{i=1}^{P}\left\|\Delta_{i}^{d}\left(\mathbf{Y}_{b}\right)\right\|^{2}$, for directions $d=\{h, v\}$, respectively, with $P$ the number of pixels of $\mathbf{Y}_{b}$. Once these values are estimated, the values for $v_{k k^{\prime}}$ were selected as $v_{k, k^{\prime}}=C /\left\|\mathbf{Y}_{k}-\mathbf{Y}_{k^{\prime}}\right\|^{2}$ where $C$ was chosen to maximize the PSNR of the obtained reconstruction. Following this procedure we obtained $\alpha^{h}=$ $\{0.13,0.1,0.07,0.1\}^{\mathrm{t}}, \alpha^{v}=\{0.13,0.11,0.07,0.11\}^{\mathrm{t}}$ and $\left\{v_{12}, v_{13}, v_{14}, v_{23}, v_{24}, v_{34}\right\}=$ $\{1.031,0.0001,0.005,0.0001,0.03,0.0001\}^{\mathrm{t}}$. 


\subsubsection{DCK}

This method requires the variographic analysis of the multispectral and panchromatic images. The experimental simple variograms of the different bands of the multispectral and panchromatic images are shown in figure 4, as well as the cross-variograms between these images, which are shown in figure 5. A linear model of coregionalization with two superimposed exponential structures has been used: a short-range one $(45 \mathrm{~m})$ and a long-range one $(728 \mathrm{~m})$. The practical ranges are $135 \mathrm{~m}$ and $2184 \mathrm{~m}$, respectively. The sills of the simple and cross-variograms of the multispectral and panchromatic bands at point support level have all been calculated using a process of numeric deconvolution and a fitting of weighted squared minima. The models induced upon pixel support can be compared with the experimental variograms and cross-variograms as shown in figure 4 and 5 (solid line).

\section{[Insert Figure 4. Approximately here]}

\section{[Insert Figure 5. Approximately here]}

Fusion by Downscaling Cokriging has been done using two bands in every case: the band whose spatial resolution is wished to be improved, and the panchromatic one. The results of the cokriging system provided the weights that were applied to the high and low spatial resolution images; that is, the multispectral and panchromatic ones, respectively. After different tests we adopted neighbourhoods of $3 \times 3$ pixels and $5 \times 5$ pixels for low and high spatial resolution images respectively.

\subsubsection{Wavelet based Methods}

\subsubsection{WAT}

In order to apply the Wavelet à Trous fusion (section 2), a fusion ratio of 4:1 between the degraded multispectral Landsat image $(120 \mathrm{~m})$ and the degraded panchromatic image $(30 \mathrm{~m})$ has been considered. Two levels of degradation have been applied to the multispectral image, so two sets of wavelet coefficients have been obtained, one containing the detail between $120 \mathrm{~m}$ and $60 \mathrm{~m}$ and the other, the detail between $60 \mathrm{~m}$ and $30 \mathrm{~m}$ (Gonzalez-Audicana et al. 2005, Lillo-Saavedra and Gonzalo 2006).

\subsubsection{AWLP}

Since the AWLP method (Otazu et al. 2005) follows the same steps than the WAT method, two levels of wavelets decomposition were applied to the panchromatic image. Bicubic interpolation was used to upsample the degraded multispectral Landsat image $(120 \mathrm{~m})$ to the size of the degraded panchromatic image $(30 \mathrm{~m})$.

\subsubsection{MDMR}

Following Lillo-Saavedra and Gonzalo (2007), in order to establish directionality and the optimal filter parameters, a large number of experiments have been carried out applying different levels of degradation $\left(1=2^{1}, 2^{2}, 2^{3}, 2^{4}\right)$ for different combinations of $a$ and $b$ (see section 2). In order to obtain the best fusion quality and a balance between 
the spatial and spectral resolution of the fused image, the optimum values of the filter parameters ( $a$ and $b$ ) have been determined by means of a simulated annealing procedure. Nonetheless, the values of $a$ and $b$, together with the number of directional filters, can be fitted to highlight the spectral or the spatial resolution so as to attain lower ERGAS values (see figure 3).

\section{[Insert Figure 3. Approximately here]}

\subsection{Image Quality Assessment}

\subsubsection{Qualitative Assessment}

In order to facilitate the qualitative evaluation of the results, two subsectors within different environmental contexts were chosen for detailed evaluation (figures 1 and 6). In sector A, the northern part is occupied by urban structures mainly, and the southern part by irrigated crops. Sector B is characterized by a variety of natural land covers, with some distinctive vegetation areas around a central water body. Figure 6 shows ETM+ 321 (RGB) true colour compositions of the five merged images along with the original multispectral image for subsectors A and B.

\section{[Insert Figure 6. Approximately here]}

For all the studied cases, the bicubic interpolation produces over-smoothed reconstructions. The fused images show considerable differences in visual quality depending on the technique applied. The FIHS, WAT, AWLP, MDMR and, specially, PCA methods have a negative impact on the colour of the image, decreasing contrast, increasing colour saturation and introducing chromatic distortions, which are associated with a decrease in the spectral quality of the image. The FIHS and wavelet methods achieve a better spatial detail and give rise to sharper images, reflected most notably in the linear features present in the urban zones (sector A). However, the greater the spatial enhancement the greater the spectral distortions and moreover, contrast is reduced, and an effect of radiometric homogenization is produced, which causes a loss of texture (Zhang and Hong 2005). This effect is particularly appreciable in the fused image obtained using the MDMR method, where the crop fields areas seem to be blurred and nearly imperceptible in some sectors (figure 6, sector B). The good performance of the Bayesian (L1COR) and the geostatistical (DCK) methods is noteworthy, which outperforms bicubic interpolation. The L1COR and DCK are the methods that best preserves contrast, saturation and texture with respect to the original reference image, showing very similar results.

\subsubsection{Direct quantitative Assessment}

\subsubsection{Assessment of the overall Quality}

In order to quantify the spectral and spatial quality of the fused images several statistical parameters were derived from the merged and the reference image (table 1): correlation coefficient and RMSE band by band, ERGAS, Q index, SSIM and the HFC (see section $3)$. The spectral indexes have been calculated using the true multispectral Landsat 
ETM+ image $\left(\mathrm{M}_{\mathrm{h}}\right)$, and the spatial indexes using the panchromatic image $\left(\mathrm{P}_{\mathrm{h}}\right)$ (see figure 2).

In general, correlation coefficients indicate that a relatively large correlation in the near infrared (NIR) band exists for all the fusion methods analysed (from 0.897 to 0.963 ) (see table 1). Correlations are however considerably higher for the green (from 0.935 to 0.966) and red visible (from 0.946 to 0.972 ) bands due to a better spectral correspondence with the panchromatic band. The enhancement of the blue may be accompanied by a severe loss of correlation in FIHS, AWLP, WAT and MDMR, due to the fact that the blue visible part of the spectra has a minor weight in the panchromatic band. With regard to the performance of image fusion methods, PCA presents the lowest correlation coefficients for the blue and green visible bands (0.881 and 0.935) and FIHS for the red and near infrared bands (0.946 and 0.897). MDMR also presents very low correlation coefficients for all the bands (from 0.890 to 0.951 ). In contrast, the DCK and L1COR methods offered the best correlation with the original multispectral image (from 0.955 to 0.972 and 0.953 to 0.971 , respectively). Table 1 shows that the RMSE are small for the DCK and L1COR methods, while the rest of the algorithms present larger RMSE values, especially the classical methods. The correlation of the high frequency components (HFC) (table 1) shows that wavelet based methods

(WAT, MDMR and FIHS, mainly) incorporate more information from the panchromatic image than DCK and L1COR methods. L1COR method provides the lowest values for this measure, in special for the blue and green bands whose correlation coefficients are equal to 0.833 and 0.837 respectively.

\section{[Insert Table 1. Approximately here]}

Low ERGAS values and high Q and SSIM index values are indicative of merged images of good quality. In a hypothetical case, an optimal result of the fusion process would yield ERGAS and Q and SSIM values equal to 0 and 1, respectively. According to the authors, an ERGAS value greater than 3 corresponds to fused products of low quality, while an ERGAS value lower than 3 denotes a product of satisfactory quality or better (Wald et al. 1997). With regard to the previous idea, DCK method, generate good quality merged images. Both DCK and L1CORclearly outperform the rest of methods according to the ERGAS index (table 2). It is worth noting that the results obtained by the classical methods, PCA mainly (12.067) are very unsatisfactory.

\section{[Insert Table 2. Approximately here]}

To ease the comparison of the different fusion methods according to the average $\mathrm{Q}$ parameter $\left(\mathrm{Q}_{\mathrm{AVG}}\right)$, we have displayed the $\mathrm{Q}_{\mathrm{AVG}}$ values for different sliding size windows. Figure 7 shows the average Q values for each fused image and for the resampled original image using bicubic convolution (BIC). All the fusion methods result in improved spectral quality with respect to the BIC image. The DCK method yields the best results with an average Q equal to 0.962. On the contrary, PCA and FIHS gave as a result the worst values of the average $\mathrm{Q}$ index ( 0.877 and 0.919 , respectively). Additionally, a comparison among algorithms, in terms of band to band $\mathrm{Q}$ index, has been carried out. All the methods yielded the poorest $\mathrm{Q}$ value for the blue band, in special the PCA, FIHS and MDMR methods (0.844, 0.857 and 0.887 , respectively). However, both DCK and L1COR methods present a much lower reduction than the 
remaining methods. The Red band was the best enhanced band for most of the methods and the DCK method obtained the best results for all the bands (from 0.955 to 0.972). The band by band SSIM index shows a different performance for every method, with the L1COR, DCK and AWLP presenting better results than the others. DCK gives higher values for band NIR, while L1COR obtains higher values for Red band, obtaining similar results in the other bands. In the average, DCK obtains the best result, with a slightly higher value than L1COR. The L1COR method gives its worst results for the NIR band and the rest of the methods, for the Red one. The average SSIM index offers very similar results to ERGAS and Q indexes. The ranking of methods confirms that wavelet are better than classical methods, with WAT and AWLP being superior to MDMR, and both the DCK and the L1COR methods yielding the best results according to the spectral indexes.

\section{[Insert Figure 7. Approximately here]}

\subsubsection{Coherence Assessment}

Another aspect of interest considered in this study is the coherence between the original image $\left(\mathrm{M}_{1}\right)$ and those estimated by the fusion algorithm once degraded to the original resolution 1 (see figure 2). A "coherence measure" has been elaborated based on: the C and RMSE of each band estimated with respect to its corresponding multispectral band (table 3). According to the previous idea, the DCK method is the most coherent, as it presents a maximum $C$ (practically equal to 1) and it minimizes the RMSE (lower than 0.8 ) for all the bands. The rest of methods have lower correlation coefficients, and higher RMSE. Classical methods present higher correlation coefficients than the rest, although root mean square errors are significantly higher too, which make them less coherent. L1COR and WAT methods are significantly less coherent than AWLP and MDMR. The high RMSE value for L1COR can be explained because it reduces the noise in the pansharpened image while incorporating the details of the panchromatic image.

\section{[Insert Table 3. Approximately here]}

\subsubsection{Indirect quantitative Assessment}

In some remote sensing applications, it is required that the fused products are susceptible of generating maps of certain accuracy (e.g., land cover/ land use classification, change analysis, etc.). To this end, the ISODATA unsupervised classification procedure has been applied to the fused images and the reference image (without downscaling) (Lillesand et al. 1987). This procedure does not require human intervention that might bias classification and determine the differentiability among spectral classes.

The high resolution multispectral image has been selected as the reference for the assessment of the accuracy of classifications of the fused images, since it represents a maximum spatial and spectral resolution for the case study. A level of quality comparable to the one derived from the classification of the original image could only be attained through the application of ideal fusion algorithms which would maximize the separability potential among classes. 
Figure 8 shows the unsupervised classifications of the original and the merged images using the analysed fusion methods. A visual inspection of the classified images suggests that the spatial distribution of the spectral classes varies notably depending on the method being used. In the classification of the original image (figure 8), six classes can be distinguished: classes 1 and 2 correspond to densely vegetated areas (irrigated land and natural vegetation, respectively); the rest of the classes correspond to non-vegetated zones or zones of scarce vegetation of high reflectivity, which range from urban areas (represented by class 3 mainly) to bare soils (class 6), including unirrigated farmlands and olive orchards (classes 4 and 5).

\section{[Insert Figure 8. Approximately here]}

Classification accuracy has been assessed by calculating the confusion matrix and the Kappa index (Congalton 1991, Congalton and Green 1999). The accuracy assessment indicated that the results corresponded to the images integrated using the L1COR, DCK and WAT methods are similar, with Kappa values equal to 0.78, 0.76 and 0.73 , respectively. For the MDMR, AWLP, PCA and FIHS images, map accuracy can be considered low, with Kappas of 0.44, 0.45, 0.64 and 0.69. In the classifications obtained using these methods approximately half of the pixels were assigned to a category matching the classification of the original image.

\section{[Insert Table 4. Approximately here]}

A detailed accuracy assessment per category indicates that classes with high reflectivity values (Class 3 to 5) present the lowest Kappa per categories values for all the classifications of PCA, FIHS, L1COR, DCK and MDMR images. For these methods, classes 3 (urban areas) and 4 and 5 (unirrigated farmlands and olive orchards) of the reference image are those presenting lesser correspondence with the merged images. In the case of classification of the AWLP and MDMR fused images, it has not been possible to differentiate between the classes of vegetation 1 and 2 .

\section{Conclusions}

Several image fusion methods of different philosophies have been compared in order to assess their performance for the integration of the panchromatic and multispectral bands of the Landsat $7 \mathrm{ETM}+$ sensor. This analysis has been carried out from diverse perspectives. On the one hand, the visual quality of the merged images has been qualitatively evaluated. On the other hand, the spatial and spectral quality, the coherence of the merged images and the mapping accuracy of the derived products from the merged images have been quantitatively assessed.

The assessment of the global quality of all merged images has demonstrated that all the methods compared produce images of a better spectral quality than classical approaches (PCA and FIHS). The algorithms based on Geostatistic (DCK) and the Bayesian algorithm (L1COR), yield better spectral results than the wavelet based fusion methods (WAT, AWLP and MDMR). These wavelet methods enhance better the spatial details of certain zones presenting specific patterns, such as the reticulate pattern of urban zones. However, they introduce some colour distortions and more homogeneous areas, such as natural vegetation or crop fields, become visually blurred. 
The analysis of the values of the following parameters: C, RMSE, ERGAS, Q and SSIM, shows that the DCK method, is the algorithm that best preserves the multispectral information of the reference image. The WAT and MDMR methods has been the most efficient in order to increase the spatial resolution of the image (as indicated by the correlation of the high frequency components). Finally, from the point of view of the joint assessment of both the spectral and spatial indexes, DCK is the method that presents the most balanced results.

DCK is the most coherent method of those analysed in this paper, since it does not introduce artefacts in the estimation of the digital numbers. Thus, the fused image, once degraded to its original resolution, is identical to the original multispectral image. However it is computationally complex, requiring variographic analysis and the fitting of simple and cross-covariance models.

In terms of mapping accuracy and considering the ISODATA classifier, the algorithms generating the images of the highest spectral quality are L1COR and DCK, with L1COR producing slightly superior results in the classifications. Future works should examine the influence of both the spatial and spectral quality of fused images using different classification algorithms (e.g. machine learning and object oriented classifiers) and in scenes of different environmental characteristics.

To sum up, we conclude that the Downscaling Cokriging and L1COR methods are efficient for increasing the spatial resolution of Landsat 7 ETM+ satellite images while maintaining the spectral contents. Therefore, the preferred use of one or the other will depend on the specific characteristics of the application in question.

\section{Acknowledgements}

We are grateful for the financial support given by the Spanish MICINN (CGL201017629 and CGL2010-15498) and Junta de Andalucía (Group RNM122). This work has been also partially supported by the "Comisión Nacional deCiencia y Tecnología" under contract TIN2010-15137 and the Consejería de Innovación, Ciencia y Empresa of the Junta deAndalucía under contracts P07-TIC-02698. The wavelet methods WAT and MDMR have been applied using the framework IJFUSION, which has been developed by the Remote sensing Group at the Faculty of Computer Science, Universidad Politécnica de Madrid.

\section{References}

AIAZZI, B., ALPARONE, L., BARONTI, S., and GARZELLI, A., 2002, Context-driven fusion of high spatial and spectral resolution images based on oversampled multiresolution analysis. Geoscience and Remote Sensing, IEEE Transactions on, 40, 2300-2312.

AlPARONE, L., WALD, L., CHANUSSOT, J., THOMAS, C., GAMBA, P., and BRUCE, L. M., 2007, Comparison of Pansharpening Algorithms: Outcome of the 2006 GRS-S Data-Fusion Contest. Geoscience and Remote Sensing, IEEE Transactions on DOI - 10.1109/TGRS.2007.904923, 45, 3012-3021.

AMOLINS, K., ZHANG, Y., and DARE, P., 2007, Wavelet based image fusion techniques - An introduction, review and comparison. ISPRS Journal of Photogrammetry and Remote Sensing, $62,249-263$. 
ATKINSON, P. M., PARDO-IGUZQUIZA, E., and CHICA-OLMO, M., 2008a, Downscaling Cokriging for Super-Resolution Mapping of Continua in Remotely Sensed Images. Geoscience and Remote Sensing, IEEE Transactions on, 46, 573-580.

ATKINSON, P. M., WEBSTER, R., and CURRAN, P. J., 1992, Cokriging with ground-based radiometry. Remote Sensing of Environment, 41, 45-60.

ATKINSON, P. M., WEBSTER, R., and CURRAN, P. J., 1994, Cokriging with airborne MSS imagery. Remote Sensing of Environment, 50, 335-345.

BUCHANAN, M. D., and PENDERGRASS, R., 1980, Digital image processing: can intensity, hue and saturation replace red, green and blue? Electro-optical systems designs, 12, 29-36.

CARPER, W. J., LILLESAND, T. M., and KIEFER, R. W., 1990, The Use of Intensity-Hue-Saturation Transformations for Merging SPOT Panchromatic and Multiespectral Image Data. Photogrammetric Engineering \& Remote Sensing, 56, 459-467.

CONGALTON, R., 1991, A review of assessing the accuracy of classifications of remotely sensed data. Remote Sensing of Environment, 37, 35-46.

CONGALTON, R. G., and GREEN, K., 1999, Assessing the Accuracy of Remotely Sensed data: Principles and Practices: CRC Press).

CHAVEZ, P. S., SIDES, S. C., and ANDERSON, J. A., 1991, Comparison of three different methods to merge multiresolution and multispectral data: Landsat TM and SPOT panchromatic. Photogrammetric Engineering \& Remote Sensing, 57, 295-303.

CHIBANI, Y., and HOUACINE, A., 2002, The joint use of IHS transform and redundant wavelet decomposition for fusing multispectral and panchromatic images. International Journal of Remote Sensing, 23, 3821 - 3833.

CHICA-OLMO, M., and ABARCA-HERNANDEZ, F., 1998, Radiometric coregionalization of Landsat TM and SPOT HRV images. International Journal of Remote Sensing, 19, 997-1005.

EHLERS, M., 1991, Multisensor Image Fusion Techniques in Remote Sensing. ISPRS Journal of Photogrammetry and Remote Sensing, 46, 19-30.

GARGUET-DUPORT, B., GIREL, J., CHASSENY, J. M., and PAUTOU, G., 1996, The use of multiresolution analysis and wavelets transform for merging SPOT panchromatic and multispectral image data. Photogrammetric Engeenering and Remote Sensing, 62, 1057-1066.

GARZELLI, A., NENCINI, F., ALPARONE, L., AIAZZI, B., and BARONTI, S., 2004, Pan-sharpening of multispectral images: a critical review and comparison: Geoscience and Remote Sensing Symposium, 2004. IGARSS '04. Proceedings. 2004 IEEE International, p. -84.

GONZALEZ-AUDICANA, M., OTAZU, X., FORS, O., and SECO, A., 2005, Comparison between Mallat's and the 'à trous' discrete wavelet transform based algorithms for the fusion of multispectral and panchromatic images. International Journal of Remote Sensing, 26, 595-614.

GRASSO, D. N., 1993, Applications of the IHS Color Transformation for 1:24,000-Scale Geologic Mapping: A Low Cost SPOT Alternative. Photogrammetric Engineering \& Remote Sensing, 59, 73-80.

LAKSHMANAN, V., 2004, A separable filter for directional smoothing. Geoscience and Remote Sensing Letters, IEEE, 1, 192-195.

LILlESAND, T. M., KIEFER, R. W., and CHIPMAN, J. W., 1987, Remote Sensing and Image Interpretation, 1 st edn (New York: John Wiley and Sons). 
LILLO-SAAVEDRA, M., and GONZALO, C., 2006, Spectral or spatial quality for fused satellite imagery? A trade-off solution using the wavelet à trous algorithm. International Journal of Remote Sensing, 27, 1453-1464.

LILLO-SAAVEDRA, M., and GONZALO, C., 2007, Multispectral images fusion by a joint multidirectional and multiresolution representation. International Journal of Remote Sensing, 28, 4065-4079.

MALLAT, S. G., 1999, A Wavelet Tour of Signal Processing (San Diego: Academic Press).

MEMARSADEGHI, N., LE MOIGNE, J., MOUNT, D., and MORISETTE, J., 2005, A new approach to image fusion based on cokriging: The Eighth International Conference on Information Fusion.

MOLINA, R., VEGA, M., MATEOS, J., and KATSAGGELOS, A. K., 2008, Variational posterior distribution approximation in Bayesian super resolution reconstruction of multispectral images. Applied and Computational Harmonic Analysis, 24, 251-267.

NISHII, R., KUSANOBU, S., and TANAKA, S., 1996, Enhancement of low spatial resolution image based on high resolution-bands. Geoscience and Remote Sensing, IEEE Transactions on, 34, 1151-1158.

NUÑEZ, J., OTAZU, X., FORS, O., PRADES, A., PALA, V., and ARBIOL, R., 1999, Multiresolutionbased image fusion with additive wavelet decomposition. Geoscience and Remote Sensing, IEEE Transactions on, 37, 1204-1211.

OTAZU, X., GONZALEZ-AUDICANA, M., FORS, O., and NUNEZ, J., 2005, Introduction of sensor spectral response into image fusion methods. Application to wavelet-based methods. Geoscience and Remote Sensing, IEEE Transactions on, 43, 2376-2385.

P., C. J., and DELFINER, P., 1999, Geostatistics: modelling spatial uncertainty, 1 st edn (New York: John Wiley and Sons).

PARDO-IGUZQUIZA, E., CHICA-OLMO, M., and ATKINSON, P. M., 2006, Downscaling cokriging for image sharpening. Remote Sensing of Environment, 102, 86-98.

PARDO-IGUZQUIZA, E., RODRÍGUEZ-GALIANO, V. F., CHICA-OLMO, M., and ATKINSON, P. M., 2011, Image fusion by spatially adaptive filtering using downscaling cokriging. ISPRS Journal of Photogrammetry and Remote Sensing, 66, 337-346.

PELlEMANS, A. H. J. M., and JORDANS, R. W. A., R., 1993, Merging Multispectral and Panchromatic SPOT Images with espect to theRadioetric Properties of the Sensor. Photogrammetric Engineering \& Remote Sensing, 59, 81-87.

POHL, C., and VAN GENDEREN, J. L., 1998, Review article Multisensor image fusion in remote sensing: Concepts, methods and applications. International Journal of Remote Sensing, 19, 823854.

RANCHIN, T., AIAZZI, B., ALPARONE, L., BARONTI, S., and WALD, L., 2003, Image fusion-the ARSIS concept and some successful implementation schemes. ISPRS Journal of Photogrammetry and Remote Sensing, 58, 4-18.

RANCHIN, T., and WALD, L., 2000, Fusion of high spatial and spectral resolution images: the ARSIS concept and its implementation. Photogrammetric Engeenering and Remote Sensing, 66, 49-61.

RIGOL, J. P., and CHICA-OLMO, M., 1998, Merging remote-sensing images for geologicalenvironmental mapping: application to the Cabo de Gata-Níjar Natural Park, Spain. Environmental Geology, 34, 194-202. 
SHETTIGARA, K. V., 1992, A generalized Component substitution technique for spatial enhancement of multispectral images using a higher resolution data set. Photogrammetric Engineering \& Remote Sensing, 58, 561-567.

SIMONE, G., FARINA, A., MORABITO, F. C., SERPICO, S. B., and BRUZZONE, L., 2002, Image fusion techniques for remote sensing applications. Information Fusion, 3, 3-15.

TU, T.-M., LEE, Y.-C., CHANG, C.-P., and HUANG, P. S., 2005, Adjustable intensity-hue-saturation and Brovey transform fusion technique for IKONOS/QuickBird imagery. Optical Engineering, 44, 116201-116201 to 116021-116210.

VEGA, M., MATEOS, J., MOLiNA, R., and KATSAGGELOS, A. K., 2010, Super Resolution of Multispectral Images using 11 Image Models and interband correlations. Journal of Signal Processing Systems.

WALD, L., 1999, Some terms of reference in data fusion. Geoscience and Remote Sensing, IEEE Transactions on, 37, 1190-1193.

WALD, L., 2000, Quality of high resolution synthesized images: is there a simple criterion?: International Conference on Fusion of Earth Data.

WALD, L., RANCHIN, T., and MANGOLINI, M., 1997, Fusion of satellite images of different spatial resolutions: Assessing the quality of resulting images. Photogrammetric Engineering \& Remote Sensing, 63, 691-699.

WANG, Z., and BOVIK, A. C., 2002, A universal image quality index. Signal Processing Letters, IEEE, 9, 81-84.

WANG, Z., BOVIK, A. C., SHEIKH, H. R., and SIMONCELLI, E. P., 2004, Image quality assessment: from error visibility to structural similarity. Image Processing, IEEE Transactions on, 13, 600612 .

YOCKY, D. A., 1996, Multiresolution wavelet decomposition image merger of Landsat Thematic Mapper and SPOT panchromatic data. Photogrammetric Engeenering and Remote Sensing, 62, 1067-1074.

ZHANG, Y., and HONG, G., 2005, An IHS and wavelet integrated approach to improve pan-sharpening visual quality of natural colour IKONOS and QuickBird images. Information Fusion, 6, 225234. 
Table 1.Band by band values of the correlation coefficients C, Root Mean Square Error (RMSE) and high frequency correlation coefficients between the reference multispectral image and the fused images.

\begin{tabular}{|lllllllllllll|}
\hline & C & C & C & C & RMSE & RMSE & RMSE & RMSE & HFC & HFC & HFC & HFC \\
PCA & 0.922 & 0.943 & 0.946 & 0.897 & 21.736 & 15.594 & 21.918 & 24.990 & 0.978 & 0.984 & 0.989 & 0.903 \\
FIHS & 0.881 & 0.935 & 0.962 & 0.951 & 13.854 & 13.280 & 11.354 & 7.896 & 0.985 & 0.985 & 0.992 & 0.990 \\
L1COR & 0.953 & 0.966 & 0.971 & 0.948 & 4.093 & 4.638 & 6.98 & 6.04 & 0.833 & 0.837 & 0.944 & 0.958 \\
DCK & 0.955 & 0.965 & 0.972 & 0.963 & 3.963 & 4.675 & 6.796 & 4.891 & 0.933 & 0.913 & 0.951 & 0.957 \\
WAT & 0.915 & 0.952 & 0.962 & 0.941 & 5.611 & 5.425 & 8.059 & 6.086 & 0.994 & 0.995 & 0.996 & 0.997 \\
AWLP & 0.913 & 0.948 & 0.962 & 0.926 & 6.022 & 5.868 & 8.068 & 7.399 & 0.980 & 0.979 & 0.973 & 0.976 \\
MDMR & 0.89 & 0.942 & 0.951 & 0.926 & 6.675 & 5.979 & 9.16 & 6.836 & 0.997 & 0.997 & 0.993 & 0.994 \\
\hline
\end{tabular}


Table 2. ERGAS values and band by band and average values of universal quality index (Q) and structural similarity index (SSIM) between the reference multispectral image and the fused images.

\begin{tabular}{|llllllllllll|}
\hline & ERGAS & Q & Q & Q & Q & Q & SSIM & SSIM & SSIM & SSIM & SSIM \\
& & B & G & R & NIR & AVG & B & G & R & NIR & AVG \\
PCA & 12.067 & 0.844 & 0.917 & 0.900 & 0.847 & 0.877 & 0.793 & 0.825 & 0.795 & 0.841 & 0.814 \\
FIHS & 7.135 & 0.857 & 0.913 & 0.958 & 0.947 & 0.919 & 0.779 & 0.818 & 0.828 & 0.853 & 0.820 \\
L1COR & 3.085 & 0.953 & 0.964 & 0.971 & 0.947 & 0.959 & 0.885 & 0.873 & 0.851 & 0.836 & 0.861 \\
DCK & 2.914 & 0.955 & 0.964 & 0.972 & 0.962 & 0.963 & 0.888 & 0.870 & 0.842 & 0.875 & 0.869 \\
WAT & 3.603 & 0.914 & 0.952 & 0.958 & 0.94 & 0.941 & 0.819 & 0.851 & 0.805 & 0.832 & 0.827 \\
AWLP & 3.890 & 0.908 & 0.947 & 0.962 & 0.923 & 0.935 & 0.844 & 0.872 & 0.846 & 0.833 & 0.849 \\
MDMR & 4.107 & 0.887 & 0.942 & 0.945 & 0.925 & 0.925 & 0.819 & 0.852 & 0.788 & 0.830 & 0.822 \\
\hline
\end{tabular}


Table 3. Band by band values of the correlation coefficients $C$ and Root Mean Square Error (RMSE) between the original multispectral image and the degraded fused images.

\begin{tabular}{|c|c|c|c|c|c|c|c|c|}
\hline & $\begin{array}{l}\text { C } \\
\text { B }\end{array}$ & $\begin{array}{l}\text { C } \\
\text { G }\end{array}$ & $\begin{array}{l}\mathbf{C} \\
\mathbf{R}\end{array}$ & $\begin{array}{l}\text { C } \\
\text { NIR }\end{array}$ & $\begin{array}{l}\text { RMSE } \\
\text { B }\end{array}$ & $\begin{array}{l}\text { RMSE } \\
\text { G }\end{array}$ & $\begin{array}{l}\text { RMSE } \\
\mathbf{R}\end{array}$ & $\begin{array}{l}\text { RMSE } \\
\text { NIR }\end{array}$ \\
\hline PCA & 0.963 & 0.975 & 0.976 & 0.931 & 21.220 & 14.735 & 20.804 & 24.474 \\
\hline FIHS & 0.938 & 0.968 & 0.986 & 0.988 & 12.621 & 12.114 & 9.246 & 5.964 \\
\hline L1COR & 0.926 & 0.953 & 0.92 & 0.884 & 5.11 & 5.12 & 11.564 & 8.804 \\
\hline DCK & 0.999 & 1 & 1 & 0.999 & 0.693 & 0.684 & 0.798 & 0.773 \\
\hline WAT & 0.835 & 0.888 & 0.933 & 0.879 & 7.59 & 7.958 & 9.872 & 8.289 \\
\hline AWLP & 0.965 & 0.982 & 0.989 & 0.970 & 3.543 & 3.348 & 4.177 & 4.402 \\
\hline MDMR & 0.937 & 0.974 & 0.977 & 0.963 & 4.608 & 3.74 & 5.962 & 4.469 \\
\hline
\end{tabular}


Table 4. Per-category Kappa values of the ISODATA classification of the fused images.

\begin{tabular}{|lcccccc|}
\hline \multicolumn{1}{r}{ Class Id. } & $\mathbf{1}$ & $\mathbf{2}$ & $\mathbf{3}$ & $\mathbf{4}$ & $\mathbf{5}$ & $\mathbf{6}$ \\
PCA & 0.86 & 0.83 & 0.53 & 0.49 & 0.69 & 0.73 \\
FIHS & 0.82 & 0.84 & 0.67 & 0.61 & 0.64 & 0.75 \\
L1COR & 0.79 & 0.81 & 0.75 & 0.75 & 0.76 & 0.85 \\
DCK & 0.81 & 0.78 & 0.73 & 0.72 & 0.75 & 0.85 \\
WAT & 0.76 & 0.76 & 0.7 & 0.69 & 0.71 & 0.82 \\
AWLP & 0.72 & 0.33 & 0.26 & 0.39 & 0.53 & 0.64 \\
MDMR & 0.35 & 0.18 & 0.32 & 0.44 & 0.56 & 0.69 \\
\hline
\end{tabular}




\section{FIGURE CAPTIONS}

Figure 1.Landsat 7 ETM+ false colour composition 432 (RGB) image of the study area $\left(944 \mathrm{~km}^{2}\right)$.It roughly corresponds to the Vega de Granada, Spain. Boxes A and B include two subsectors showing different land cover context used for qualitative assessment of results.

Figure 2. Flowchart of the methodology used for comparative assessment of image fusion algorithms

Figure 3. Spectral and spatial planes for level $k=2$ of the Multidirection- Multiresolution method

Figure 4. Experimental variograms and induced models of the bands corresponding to blue, green, red and near infrared, the panchromatic image (from top to bottom and left to right, respectively)

Figure 5. Cross-variograms and induced models between the multispectral bands, (blue, green, red and near infrared), and the panchromatic band (from top to bottom and left to right, respectively)

Figure 6. Details of the true colour compositions of the original multispectral image and the fused images for test sectors A and B. The following images are shown from top to bottom and left to right: Reference multispectral image, PCA fused image, FIHS fused image, L1COR fused image, DCK fused image, WAT fused image, AWLP fused image, MDMR fused image and the bicubic resample of the original multispectral

Figure 7. Graphical representation of the $\mathrm{Q}_{\mathrm{AVG}}$ values of the Landsat 7ETM+ fused images for different sliding windows sizes

Figure 8. From top to bottom and left to right: Maps derived from the classification of the original image and the fused images by PCA, FIHS, L1COR, DCK, WAT, AWLP and MDMR (from top to bottom and left to right, respectively) 
FIGURE 1

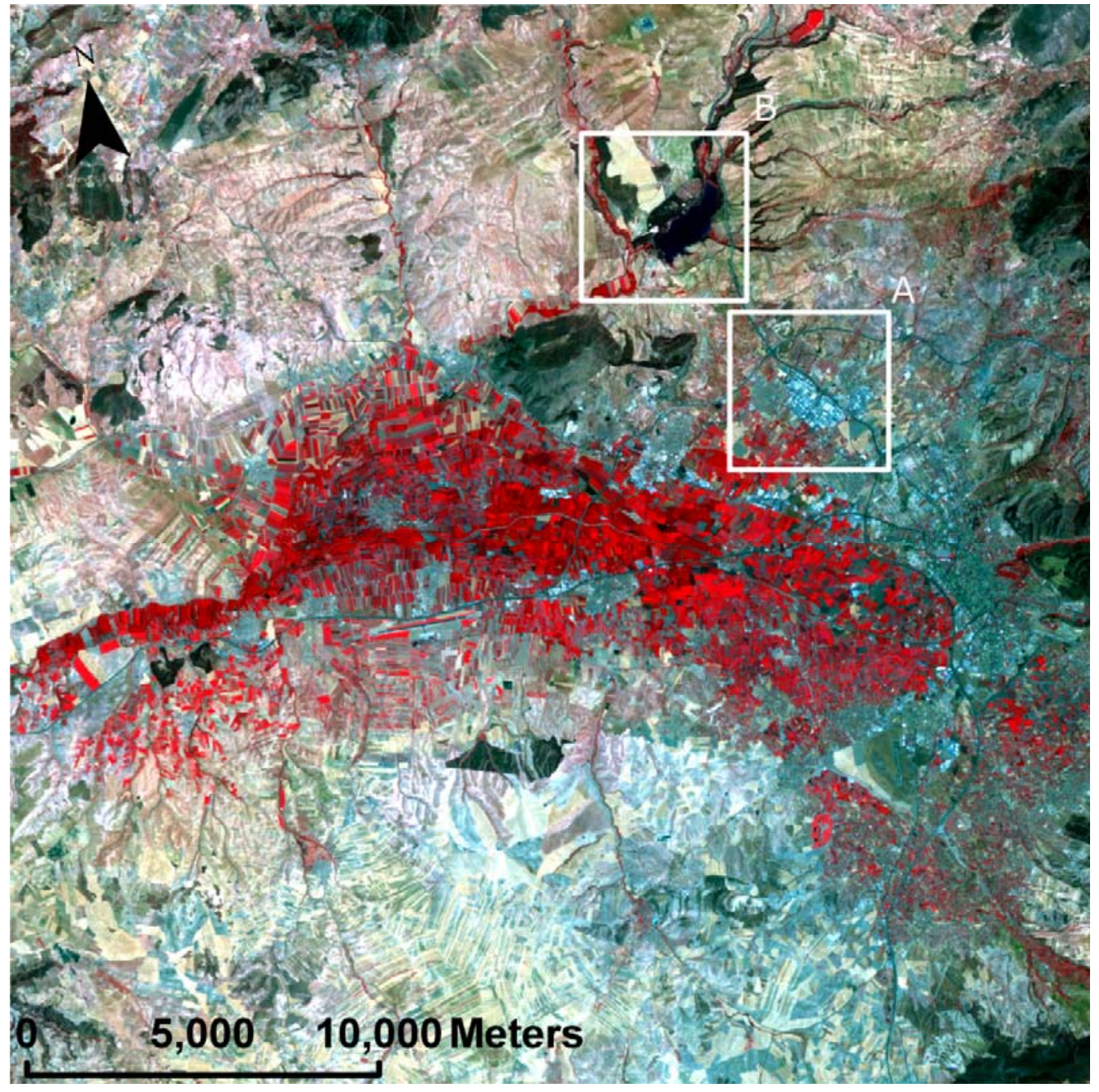


FIGURE 2

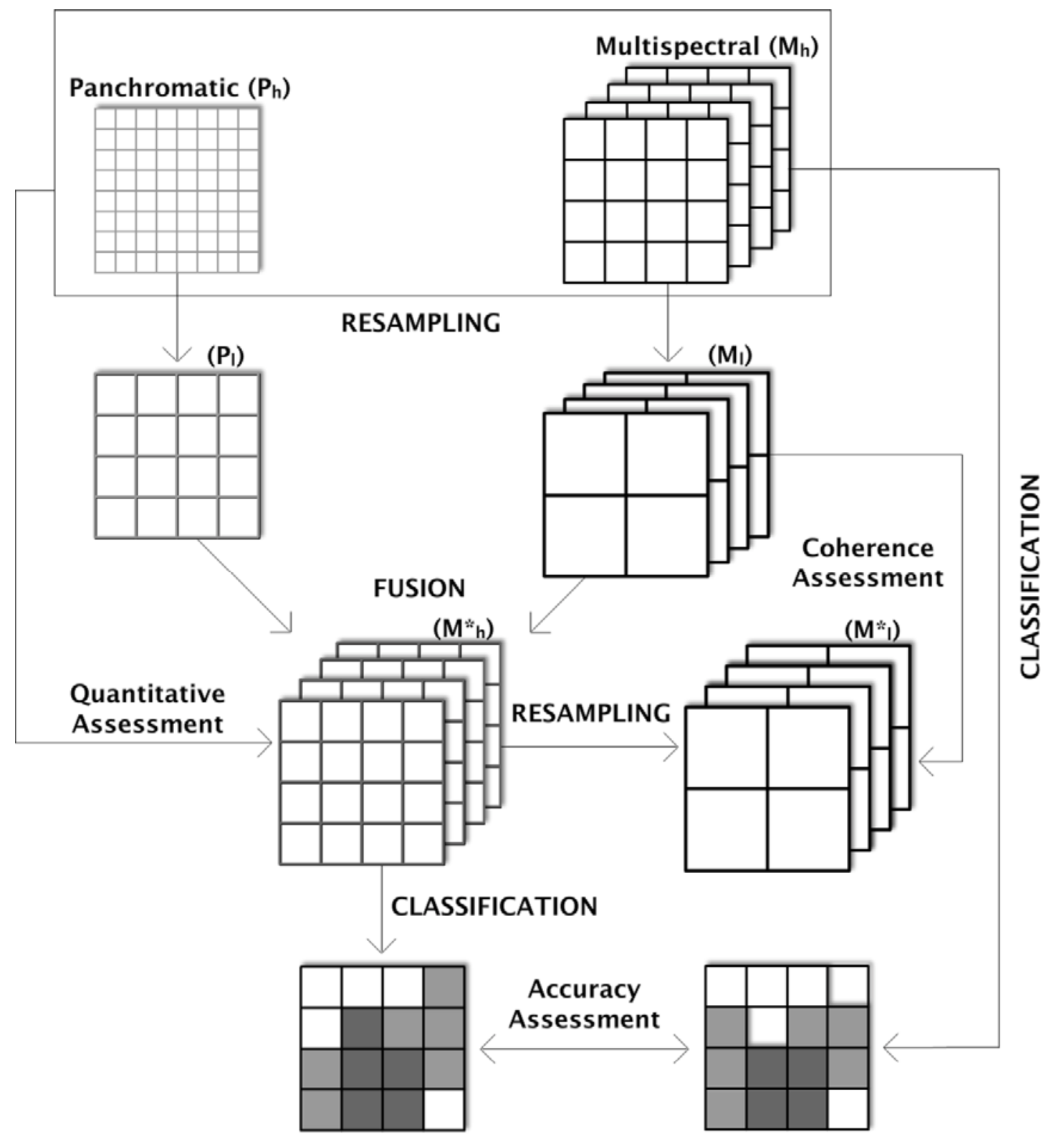


FIGURE 3

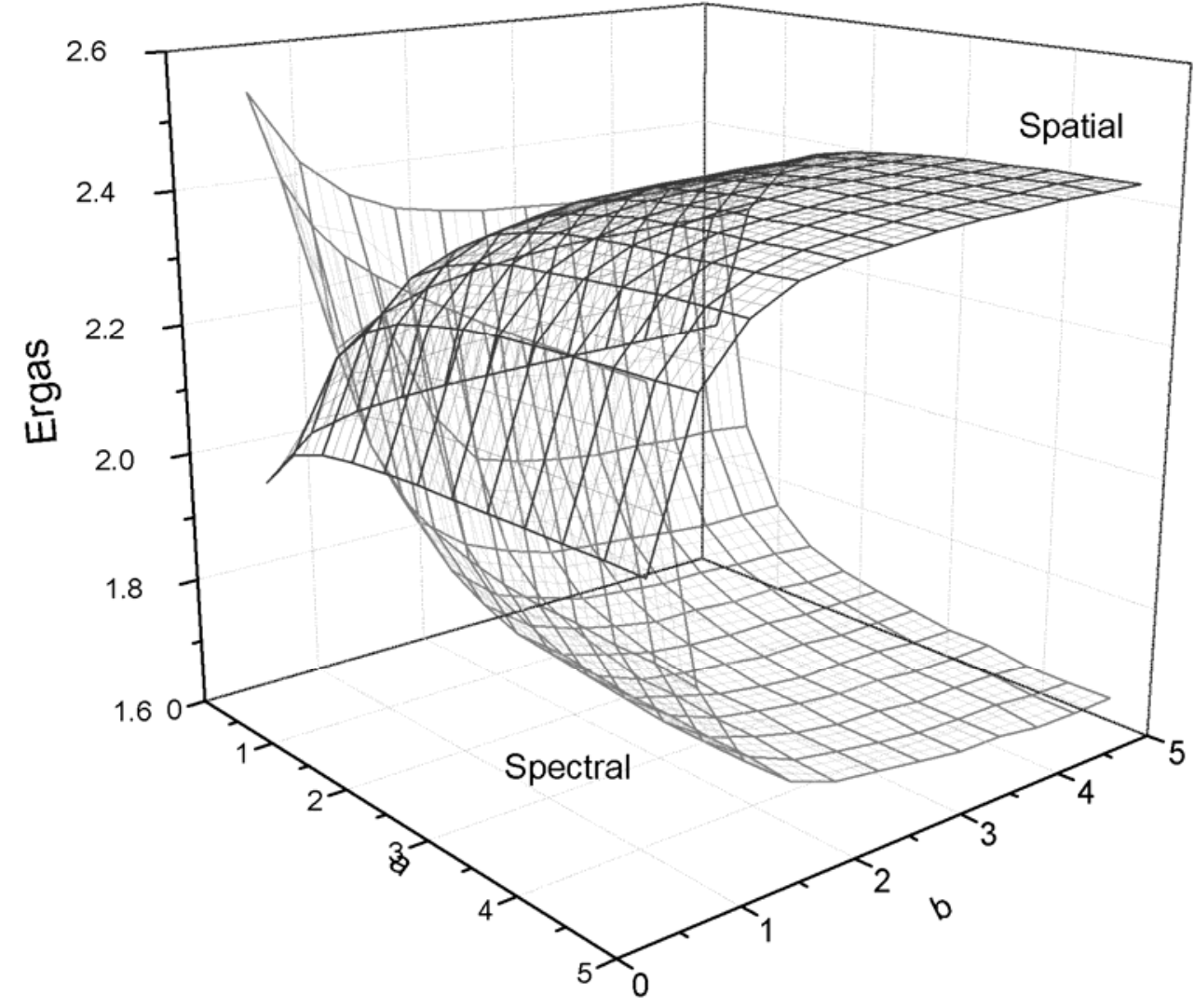


FIGURE 4
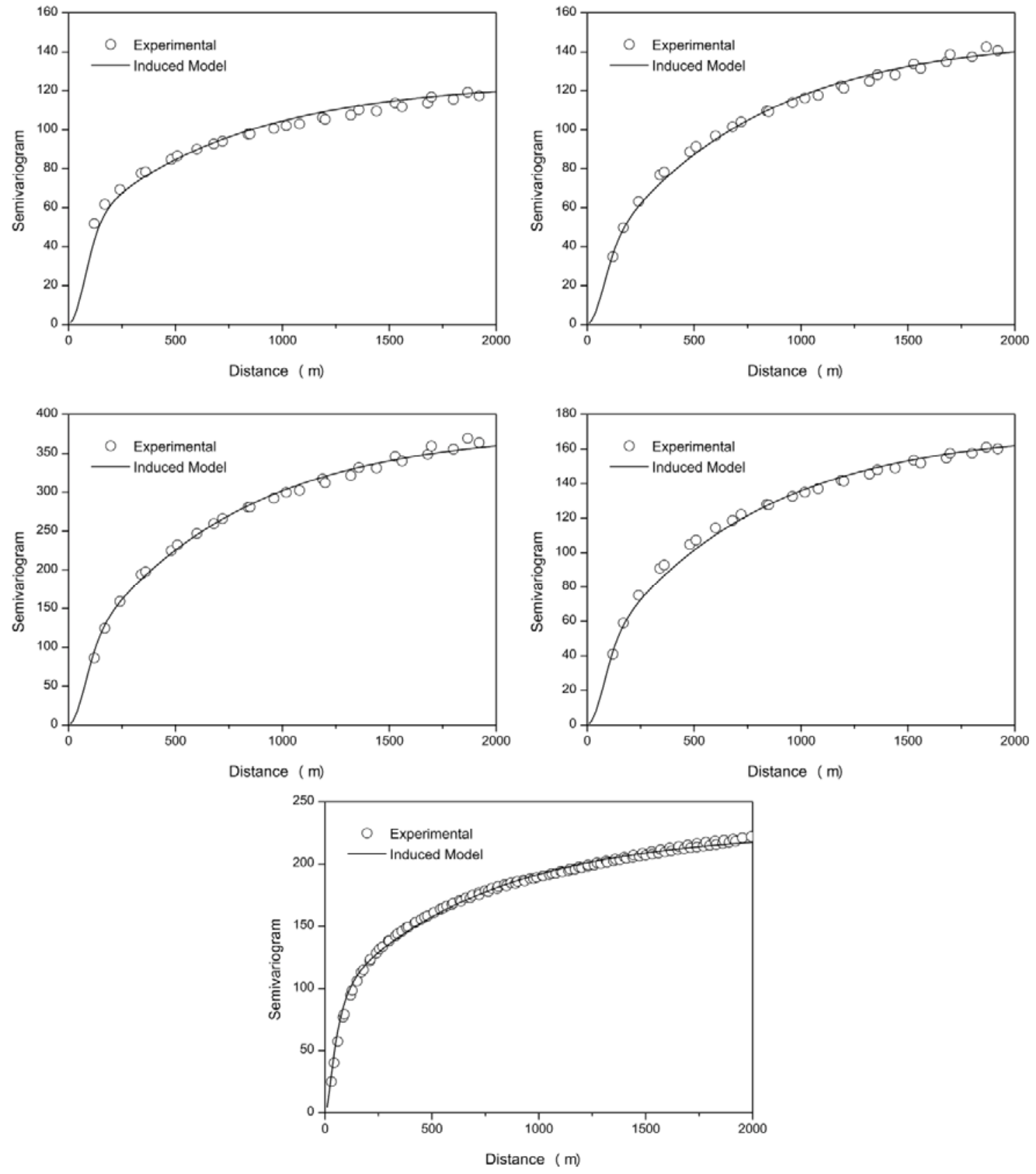
FIGURE 5
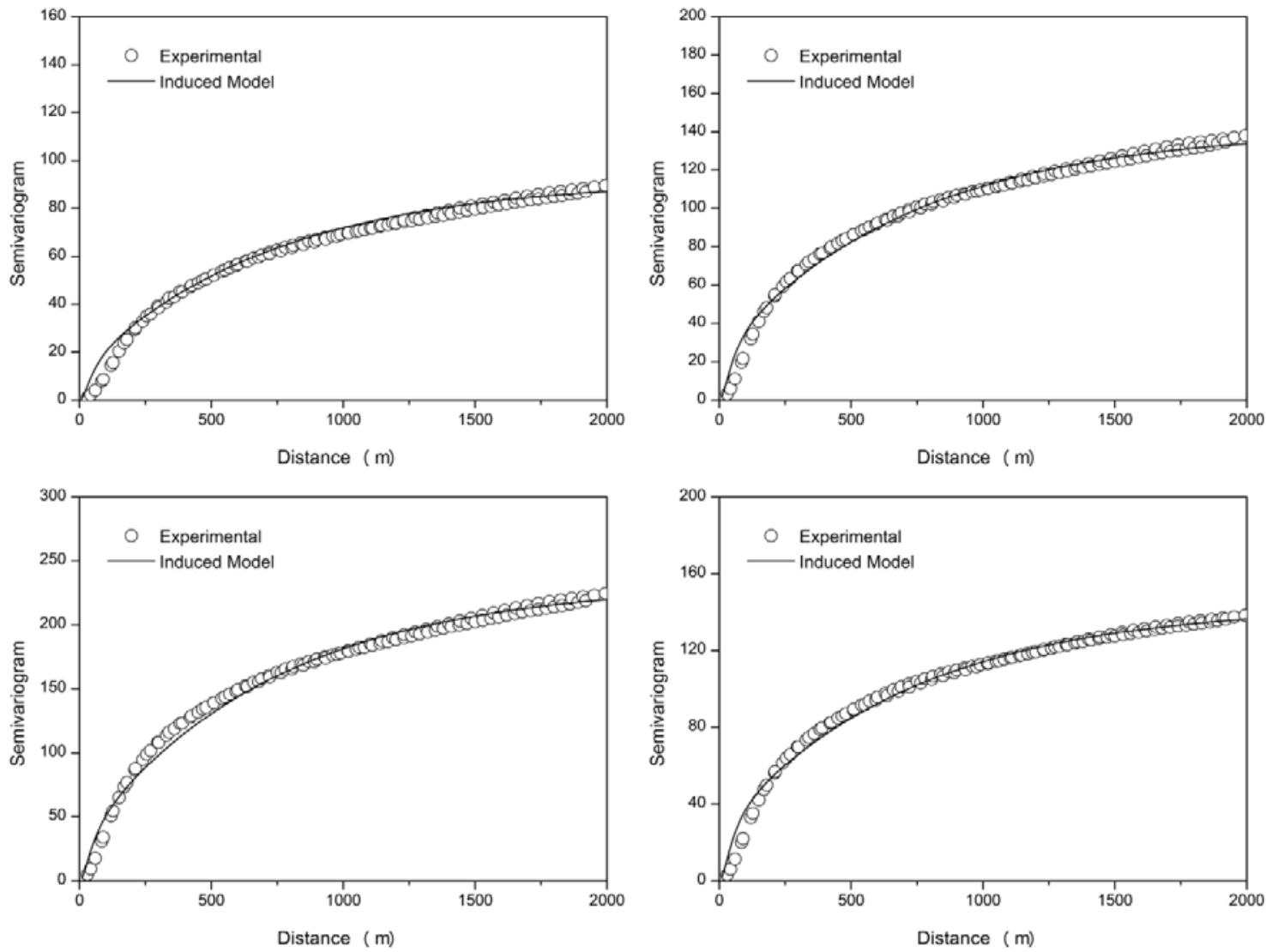
FIGURE 6

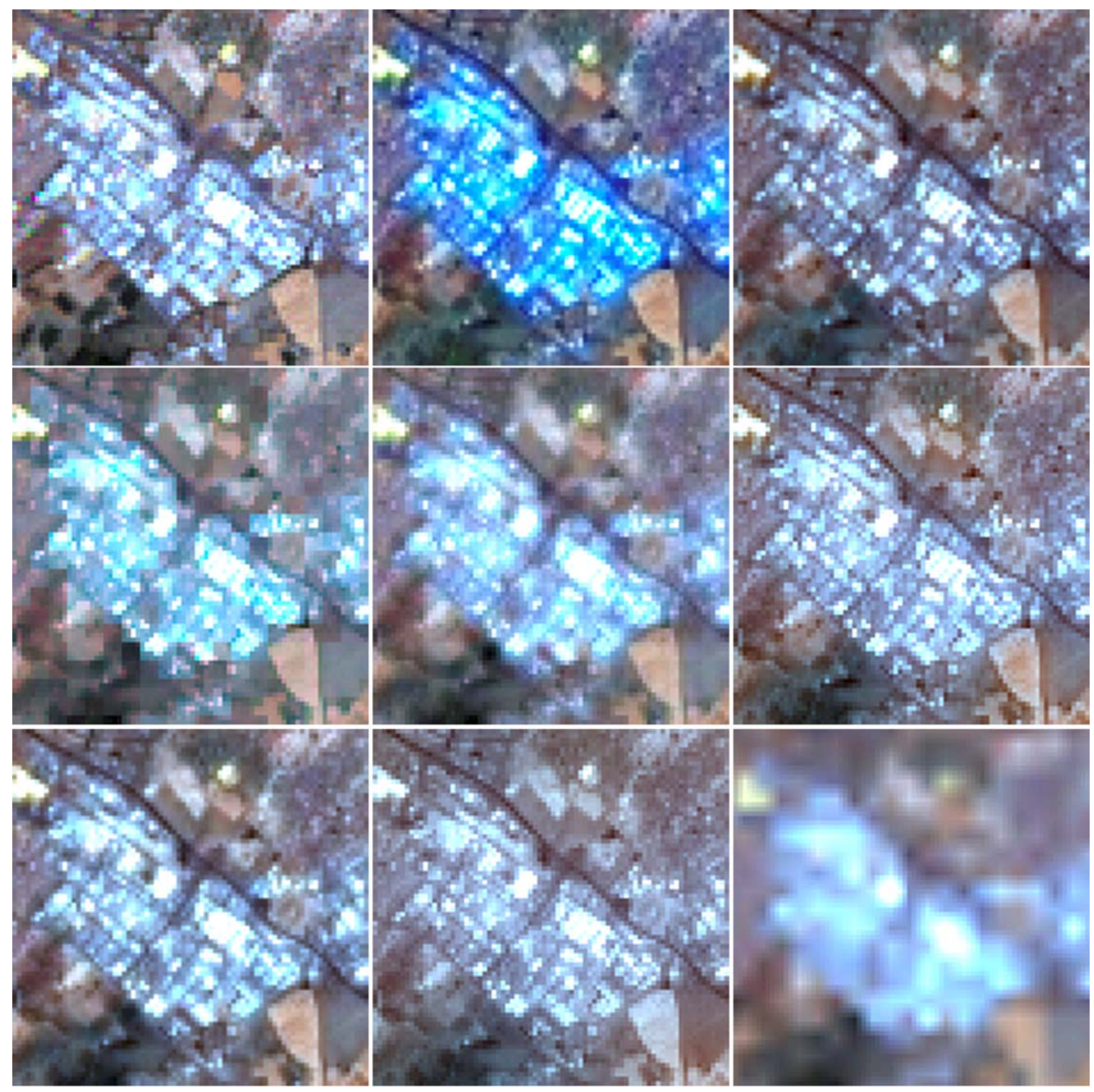


FIGURE 6 (continuation)

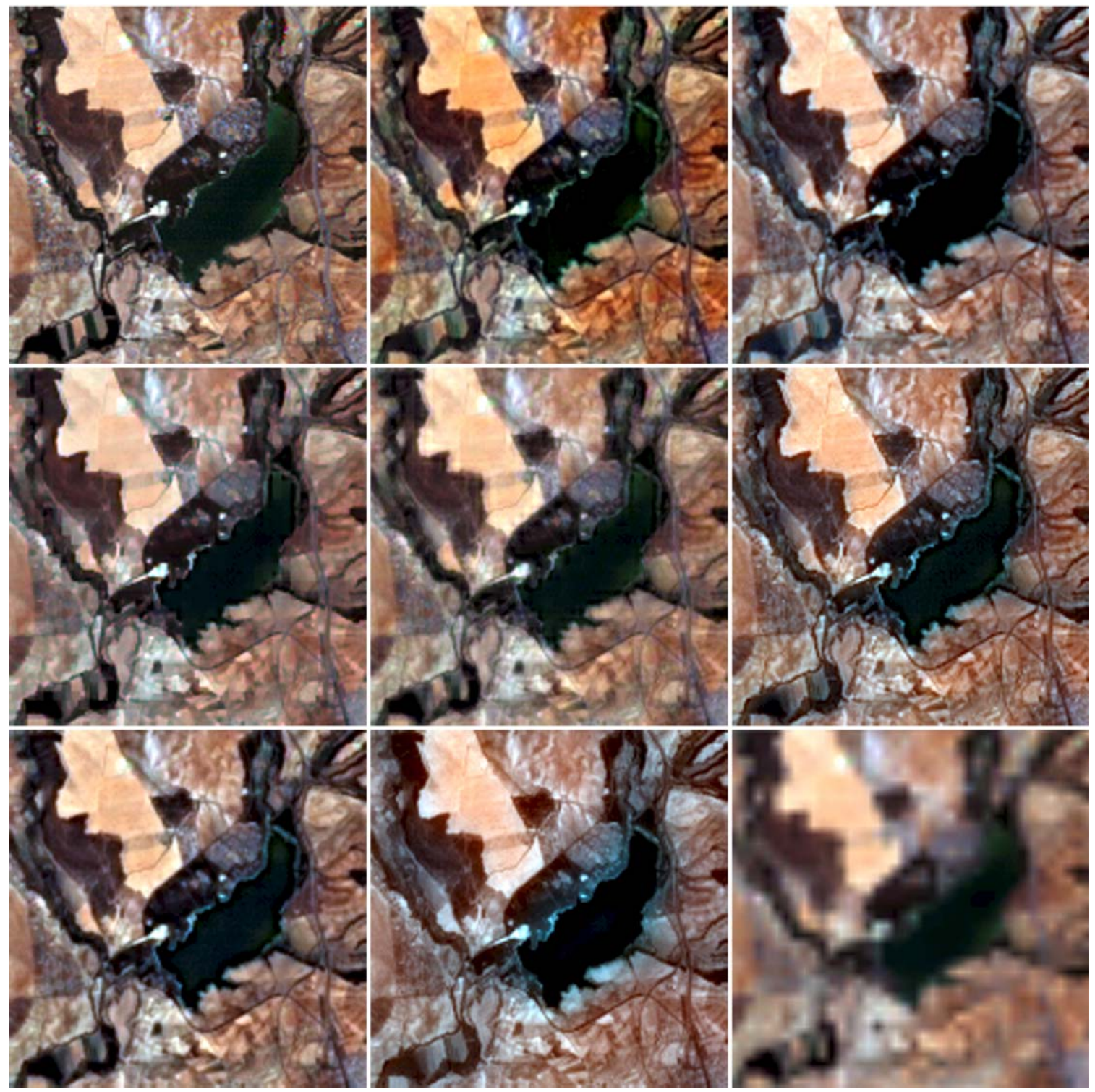


FIGURE 7

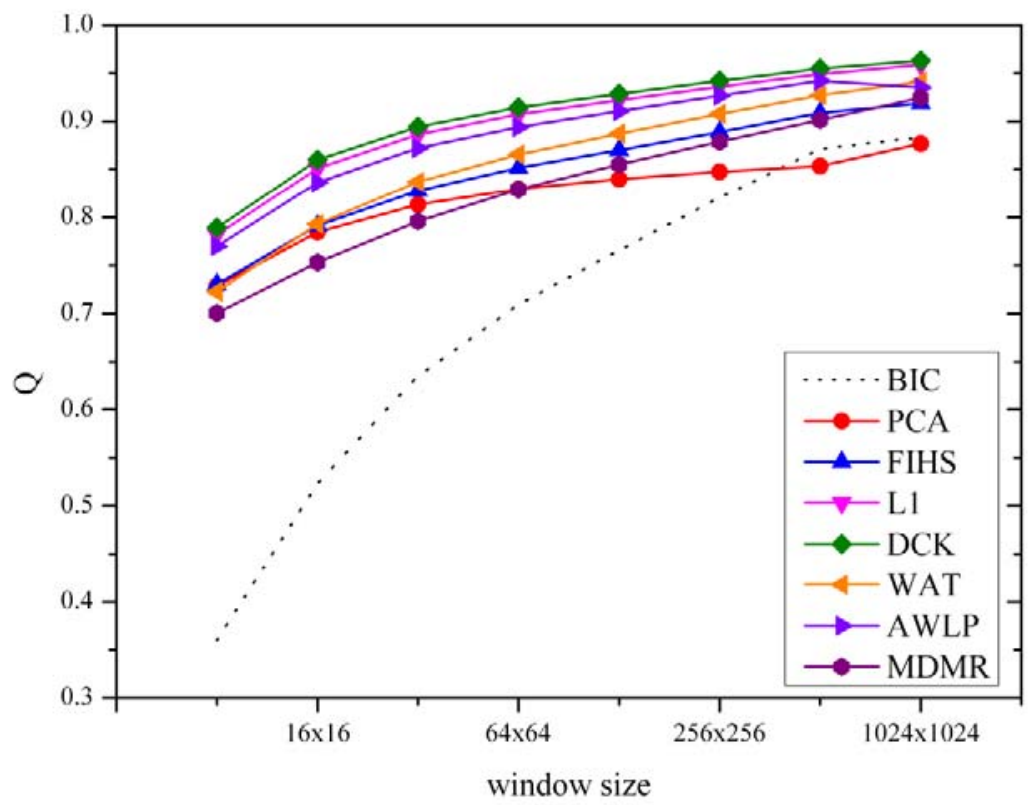


FIGURE 8
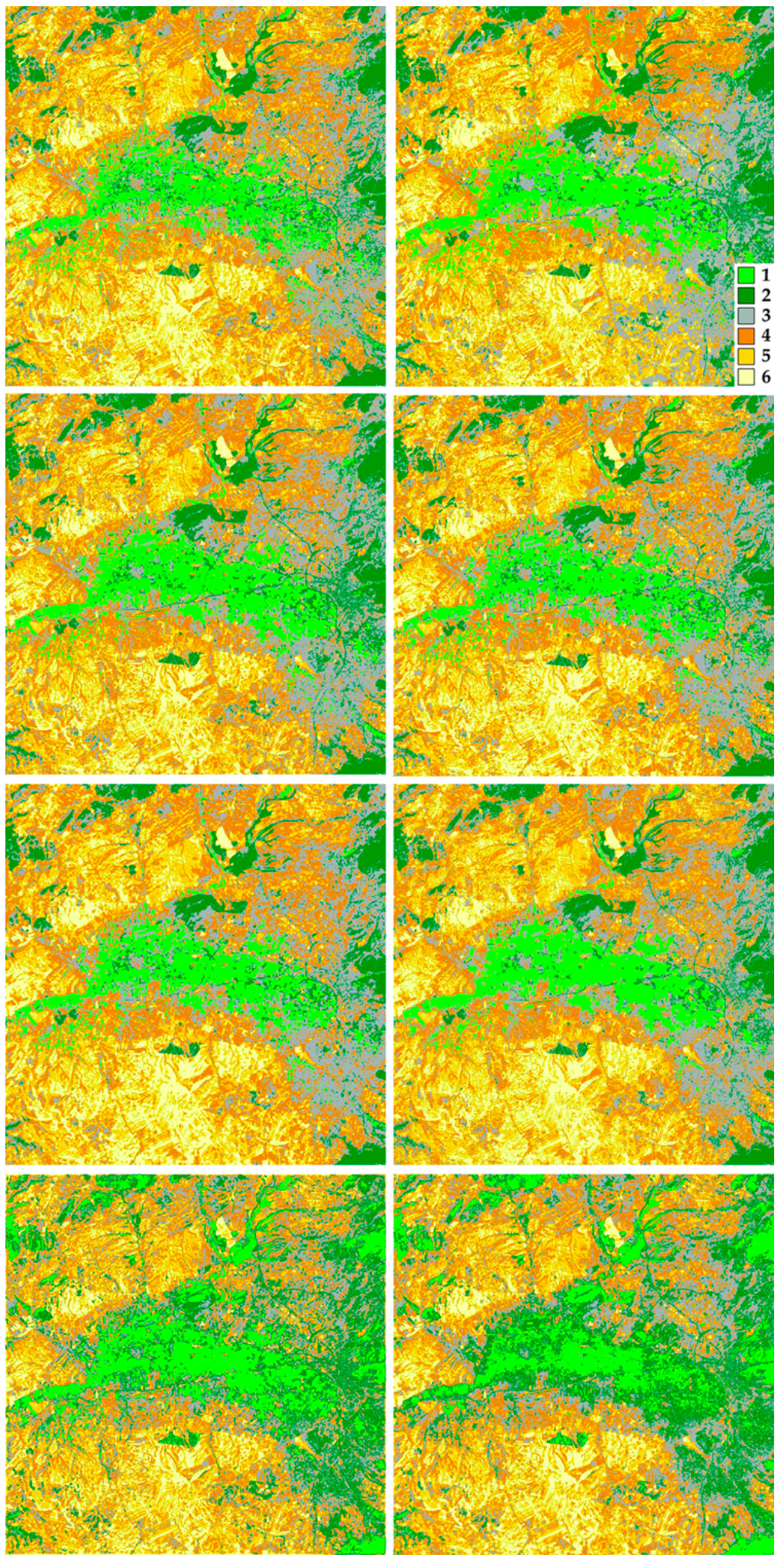